\title{
Projekt einer Siedlungsgeschichte des Kantons Zürich
}

1. Eine bereits vorhandene Vorstufe zur Arbeit Im März 1965 erteilte der damalige Vorsteher des Amtes für Regionalplanung des Kantons Zürich, H. Aregger, dem Verfasser den Auftrag für eine Untersuchung über die Entwicklung der Siedlungsstruktur im Kanton Zürich von den Anfängen der Besiedlung bis zur Gegenwart. Im Interesse der Raumplanung sollte eine Antwort auf die Frage, ob und in welchem Masse die "geschichtliche Vorformung" der Siedlungsstruktur als eine Determinante ihrer künftigen Entwicklung zu gewichten sei, gefunden werden. Der Auftraggeber setzte so der Untersuchung nicht nur ihre Ziele, er förderte die Arbeit auch unmittelbar durch eine Reihe siedlungsgeschichtlicher Begriffe, Hypothesen und Feststellungen, die er dem Verfasser mitgab. Die Untersuchung wurde bis nahe an die Gegenwart (1920) geführt und der Bericht bis Ende 1966 in drei Folgen dem Amt für Regionalplanung abgeliefert.

Auf eine Anregung von E. Winkler, Professor an der ETH, unterzog der Verfasser zusammen mit seinem Bruder Hans Litz, dipl. Arch. ETH, das Manuskript einer gründlichen Durchsicht und erstellte eine Liste von zusätzlichen Anforderungen. Sie kamen zum Schluss, dass die seinerzeit angefertigte Studie - natürlich mit Ausnahme der mangelhaften und veralteten Partien - als Materialsammlung wohl brauchbar sei, dass aber die Arbeit nun unter völlig neuen Gesichtspunkten angegangen werden müsse. Es schien zudem richtig, die Arbeit aus ihrer ausschliesslichen Bindung an die Raumplanung zu lösen und sie zu einer eigentlichen Siedlungsgeschichte des Kantons Zürich umzugestalten, wobei das Schwergewicht vom Text, der einerseits stark zu straffen, andererseits aber auch zu ergänzen wäre, auf das Optische (Karten, Pläne, Abbildungen, Graphiken) verlegt werden müsste. Aus diesen Erwägungen ging schliesslich das hier vorgelegte Projekt hervor. Der Hauptgrund für dessen Veröffentlichung in der Geographica Helvetica liegt im Erfordernis, eine interdisziplinär aus Vertretern der Geographie, Geschichte, Soziologie und Raumplanung zusammengesetzte Sachbearbeitergruppe für die projektierte umfangreiche und kom plexe Arbeit zu gewinnen. Die Bildung dieser
Arbeits gruppe ist im Gange; weitere Interessenten sind willkommen.

\section{Begründung der neu projektierten Arbeit}

\section{1. Motive}

- Mit der projektierten Siedlungsgeschichte des Kantons Zürich soll nicht in erster Linie die Rekonstruktion der Vergangenheit um ihrer selbst willen angestrebt werden.

- Hauptmotive und Ausgangspunkte sind vielmehr die aktuellen Fragestellungen der Gegenwart, wie sie in der öffentlichen Diskussion auf den Gebieten der Raumplanung, des Umweltschutzes (inkl. Ortsbildschutz), des Bodenrechts, des Wohnungswesens, der Lokalisation von Arbeitsplätzen und des Verkehrswesens zutage treten, sowie die Fragen nach der zukünftigen Besiedlung und Bodennutzung überhaupt.

- Diese Motive sollen wegleitend sein für die Auswahl des Stoffes sowie für das Vorgehen bei dessen Erforschung und Darstellung. Die Arbeit ist dabei so breit anzulegen, wie es für das Verständnis des Gesamtzusammenhanges erforderlich ist.

- Zusätzlich begründet erscheint das Projekt durch den Umstand, dass die Bau- und Siedlungstätigkeit infolge der Rezession seit 1975 stagniert sowie durch die Tatsache, dass das zürcherische Bau- und Planungs gesetz voriges Jahr in Kraft gesetzt wurde und in nächster Zukunft möglicherweise ein neues eidgenössisches Raumplanungsgesetz erlassen wird.

Eine Zäsur, wie sie durch diese Vorgänge entsteht, bewirkt besonders günstige Voraussetzungen für eine distanzierte und daher objektive Betrachtung geschichtlicher Entwicklungen von der Vergangenheit über die Gegenwart in die Zukunft.

2. 2. Zwecke

Die projektierte Siedlungsgeschichte versucht insbesondere

- der Raumplanung die historische Vorformung der Siedlungsstruktur im Kanton Zü-

Karl LITZ, Dr.phil. ,Alte Zürcherstrasse 21, 8903 Birmensdorf 
rich und damit geschichtliche Determinanten in den künftigen Entwicklungstrends aufzuzeigen,

- dem Umweltschutz (besonders dem Ortsbildschutz) zusätzliche Grundlagen und Kriterien für künftige Entscheidungen und Massnahmen zu liefern,

- der Bau- und Wohnwirtschaft durch Ausweitung des geschichtlichen Erfahrungshorizontes eine verbreiterte Einsicht in die Möglichkeit bzw. Wahrscheinlichkeit neuer Siedlungsund Wohnweisen zu verschaffen,

- als mögliches Modell und Vorstufe für eine gesam tschweizerische Siedlungsgeschichte mit analoger Zielsetzung zu dienen.

Die Siedlungsgeschichte des Kantons Zürich richtet sich daher, falls sie ausgeführt wird, an Raumplaner, Architekten, Politiker, Geographen, Historiker, Soziologen und Umweltwissenschafter - auch an die Studierenden dieser Fachrichtungen - sowie an eine breitere Oeffentlichkeit, soweit sie an den historisch gewordenen Schichtungen und Gefügen des Siedlungsbildes und an dessen künftiger Entwicklung und Gestaltung interessiert ist.

3. Provisorisches Dispositiv (ohne Feingliederung) und Kurzfassung der Arbeit

3. 1. Hauptbegriffe 1)

3. 1. 1. Räumliche Hauptbegriffe

Siedlung: eine Gesamtheit von Gebäuden (im Grenzfall ein einziges Gebäude), deren horizontale und vertikale Abstände von einander ein bestimmtes Maximum nicht übersteigen und die nicht durch unüberschreitbare Hindernisse (z. B. Gewässer, Eisenbahntrasses) von einander getrennt sind. Ansätze zur exakten Bestimmung dieser Kriterien finden sich in: $\mathrm{H}$. Windler und E. Winkler:Zur quantitativen Bestimmung von Siedlungseinheiten; Plan, 1950, Nr. 6, S. 180-189: In Abweichung vom ursprünglichen Wortsinn und von anderweitigen Definitionen umfasst der hier verwendete Begriff nicht nur Wohnstätten sondern sämtliche Bauten (auchBetriebsstätten, öffentliche Gebäude, etc.).

Siedlungselement: In einer Siedlung, die aus einer Mehrzahl von Gebäuden besteht, kann jedes Gebäude als Element betrachtet werden sowie jede Gebäudegruppe, die gegenüber den anderen Teilen der Siedlung mindestens ein unterscheidendes Merkmal aufweist. Jede Siedlung kann ihrerseits als Element der Gesamtheit der Siedlungen in einem Teilgebiet des Kantons Zürich betrachtet werden, sofern dieses Teilgebiet unter einem bestimmten (z.B. orographischen, wirtschaftlichen oder politisch-administrativen) Gesichtspunkt als Einheit erscheint. Jede solche Gesamtheit kann schliesslich Siedlungselement im Gesamtgebiet des Kantons Zürich sein.

Siedlungsstruktur: Mass und Art der Anordnung der Elemente in einer bestimmten Siedlung bzw. in einer bestimmten Gesamtheit von Siedlungen.

Siedlungssystem: eine Gesamtheit von Siedlungselementen mit geformter Struktur. Siedlungskonglomerat: eine Gesamtheit von Siedlungselementen $\mathrm{m}$ it amorpher Struktur. - Ein Siedlungskonglomerat kann in Teilgebieten Siedlungssysteme enthalten und umgekehrt.

3. 1. 2. Zeitliche Hauptbegriffe

Vorbemerkung: Der erste Griff zur Erfassung eines geschichtlichen Vorganges ist seine Aufgliederung in bestimmte Entwicklungsphasen, der zweite das Aufzeigen der Faktoren, welche dafür verantwortlich sind. Bei der Siedlungsgeschichte können beide zusammengefasst werden; weil sie zur Hauptsache von der Entwicklung der Technik determiniert wird, können die Epochen der Geschichte der Technik die Phasen der Siedlungsentwicklung bezeichnen. Dieses hier gewählte Vorgehen entspringt nicht einer monokausalen Auffassung der Geschichte; weit deutlicher, als es in einer Kurzfassung möglich ist, müsste in der ausgeführten Arbeit auf die Interdependenz zwischen technischen, wirtschaftlichen, kulturellen und politischen Entwicklungsfaktoren der Siedlungsgeschichte hingewiesen werden. An dieser Stelle sei lediglich die Korrelation zwischen Technik und Wirtschaft - die wichtigste in siedlungsgeschichtlichem Zusammenhang - knapp veranschaulicht: Es ist für die Anteile der Bevölkerung an den verschiedenen Wirtschaftssektoren und für deren Siedlungsweise entscheidend, ob die 
landwirtschaftliche Gütererzeugung bloss mit Hilfe von Geräten oder auch mittels Maschinen und unter Beizug der Agrarchemie erfolgt, ob die Güterverarbeitung vor allem mit Werkzeugen oder überwiegend mit Maschinen sich vollzieht und ob Güterverteilung und Personentransport ohne Kraftmaschinen verlaufen, ob mit der Eisenbahn oder über den motorisierten Individualverkehr. - Die Anregung für die folgenden Epochenbezeichnungen verdankt der Verfasser P. Hall: The World Cities; London, 1966 (deutsche Uebersetzung: P. Hall: Weltstädte; München, 1966), der in seiner Publikation den Begriff "Neotechnisches Zeitalter" verwendet.

Paläotechnisches Zeitalter = die Epoche des fast ausschliesslichen Werkzeuggebrauchs in sämtlichen Wirtschaftssektoren. Es ist vor allem durch das Fehlen von Kraftmaschinen gekennzeichnet (mit wenigen Ausnahmen, z.B. Mühlen). - Das paläotechnische Zeitalter wird abgeschlossen durch die technische Revolution. Die technische Revolution beginnt damit, dass die Handhabung von Werkzeugen durch die Handhabung von Maschinen ersetzt wird. Mesotechnisches Zeitalter = die Epoche der Wasserkraftmaschine sowie der Dampfmaschine als Kraftmaschine für Industrie und Verkehr.

Neotechnisches Zeitalter = die Epoche des Elektromotors und des Explosionsmotors als Kraftmaschinen für Industrie und Verkehr sowie der Elektronik für die Nachrichtenübermittlung (Radio-Elektronik) und für die Automation (elektronische Maschinen-Steuerung).

Die Gleichsetzung von "technischer Revolution", die sich dadurch vollendet, dass die Automation die Steuerung von Maschinen übernimmt, mit "industrieller Revolution" ist m.E. unzulässig, da die industrielle Produktion, d.h. die systematisch-rationelle Massenfertigung, in der Form von Hausindustrie und Manufaktur, lange vor der Mechanisierung, bereits im Spätmittelalter einsetzt (mit Vorstufen, die bis in die Antike zurückreichen).

3. 2. Die Epochen der Siedlungsgeschichte

3. 2. 1. Paläotechnisches Zeitalter (Neolithikum bis $1800 \mathrm{n}$. Ch. )

3. 2. 1. 1. Vom Neolithikum bis zum Ende der gallorömischen Epoche
Die Siedlungsgeschichte des Kantons Zürich begimt mit dem Neolithikum, d.h. mit der erstmaligen Niederlassung einer sesshaften Bevölkerung. Sie muss aber bis zum Ende der gallorömischen Epoche - mit Ausnahme von zentralen Orten (Turicum, Vitudurum) sowie von Hauptverkehrslinien - lediglich in den Grundzügen dargestellt werden, da bei den dispersen (d.h. nicht zentralen) Orten von der römischen Epoche zum Frühmittelalter überwiegend keine Siedlungskontinuität besteht. 3.2. 1.2. Die Epoche vom Frühmittelalter bis zur Mitte des 17. Jahrhunderts mit der Agrarwirtschaft als Hauptfaktor für die Entwicklung der Siedlungsstruktur

In der Zeit der alemannischen Landnahme (5. - 9. Jahrhundert) entstand der Raster der paläotechnischen Siedlungsstruktur in seinen Hauptzügen. Auf die Ursiedlungsepoche (5. und 6. Jh. ) ist die Dorflandschaft mit ihrem weitmaschigen, relativ gleichmässigen Siedlungsnetz zurückzuführen, während die Kulturlandschaften gemischter Siedlungsweise (Dörfer, Weiler, Höfe)mit entsprechend engermaschigem und weniger gleichmässigem Siedlungsnetz in die Ausbauperiode (7. -9. Jh. ) zurückreichen. Der erhebliche Bevölkerungszuwachs im Hochmittelalter erforderte ausgedehnte Rodungen. Die Rodungsperiode (10. -13. Jh. ) brachte schliesslich vor allem in voralpinen Gebieten (z. B. Tössbergland) die Regionen mit unregelmässig gestreuten Hofsiedlungen hervor. Ist das System der dispersen Orte mit dem Hochmittelalter im wesentlichen abgeschlossen, so setzt die Entfaltung der zentralen Orte in dieser Epoche erst eigentlich ein. Zürich wird 1218 Freie Reichsstadt, d.h. praktisch souveräner, politisch voll handlungsfähiger Gemeindeorganismus und durch Erwerb des Kantonsgebietes im Spätmittelalter Landesherrin eines Stadtstaates, mit Privilegien auch wirtschaftlicher Art (Monopole in Handel, Gewerbe und Industrie).

Untergeordnete Verwaltungs-, Gewerbe- und Handelszentren waren die Landstädte (am wichtigsten Winterthur). Um die Mitte des 17. Jahrhunderts hat das gerasterte paläotechnische Siedlungssystem mit den hierarchisch abgestuften zentralen Orten als Knotenpunkten seine vollendete Ausprägung erreicht. 
3. 2. 1. 3. Die Hausindustrie als zweiter Hauptfaktor für die Entwicklung der Siedlungsstruktur (1650 - 1800)

Seit der Mitte des 17. Jahrhunderts beginnt im agrarisch grossenteils benachteiligten voralpinen Gebiet des Kantons Zürich, südöstlich der klimatischen und geomorphologischen Scheidelinie Zürich-Winterthur, die Hausindustrie (besonders Baumwollspinnerei und -Weberei) siedlungsgeschichtlich sich auszuwirken. Kann man bis dahin die Wirtschaft nicht als selbständige Determinante für die Entwicklung der dispersen Struktur auffassen, denn diese Struktur war ja fast ausschliesslich von den natürlichen Determinanten Bodenform, Bodenqualität und Klima abhängig gewesen, so beginnt sich nun in den Hausindustriegebieten die Wirtschaft von der Determinante Natur zu trennen und tritt als eigenständiger Entwicklungsfaktor des Siedlungsbildes in Erscheinung. Aber trotz der dadurch erfolgenden Ausweitung der Siedlungsräume und der erheblichen Verengung des Siedlungsmaschennetzes blieb das typisch paläotechnische gerasterte Siedlungssystem erhalten, und auch die Hierarchie der Knotenpunkte, d.h. der zentralen Orte blieb wegen der Kontinuität der politischen Verfassung konstant.

3.2. 2. Mesotechnisches Zeitalter (1800-1920)

3.2.2. 1. Die Epoche der fast ausschliesslich mit Wasserkraft betriebenen Fabrikindustrie (1800- ca. 1860)

Der auf Liberalismus und Demokratie ausgerichtete Umsturz von 1798 beseitigte mit der Aufhebung der traditionalistischen und hierarchischen Verfassung des Ancien Régime eine Hauptursache für die Konservierung des paläotechnischen Siedlungssystems: Die Fabrikations-, Gewerbe - und Handelsmonopole der Städte (in erster Linie der Stadt Zürich) fielen dahin, und die alten Dorfverfassungen mit Dreifelderwirtschaft, Flurzwang und Beschränkungen der Baufreiheit lösten sich auf. Gleichzeitig begann die Mechanisierung der Industrie. Für diese Fabrikindustrie wurden durch die gleichzeitige Verbesserung der Agrartechnik Arbeitskräfte aus der Landwirtschaft freigesetzt, was die Umwandlung des Kantons Zürich vom Agrarstaat zum Industriestaat ermöglichte. Für die Veränderung der Siedlungsstruktur in der ersten Phase der mesotechnischen
Epoche war die Verwendung der Wasserkraft als Antrieb für die Fabrikindustrie von entscheidender Bedeutung. Die Fabriken und die sich an sie anschliessenden Kosthäuser und Industrieweiler reihten sich perlschnurartig an die energieergiebigen Wasserläufe zumal des südöstlichen Kantonsteils an und durchbrachen so den Raster der paläotechnischen Struktur (Abb. 1). Allerdings vermochten diese gleichsam linearen Durchbrüche das aus der paläotechnischen Epoche überkommene Siedlungsbild, im ganzen gesehen, noch nicht radikal umzugestalten.

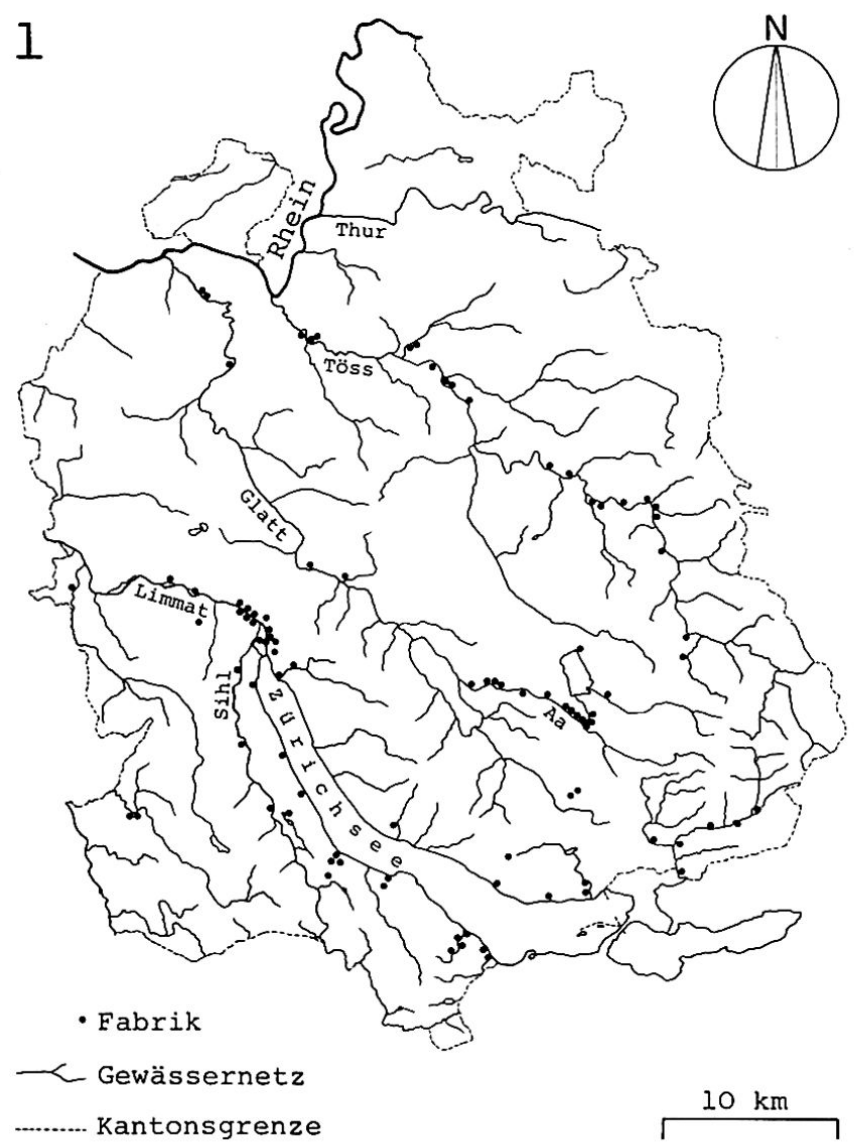

Abb. 1: Fabriken um 1850.

Quellen: Karte des Kantons Zürich im Maßstab von 1: 25000 , nach den in den Jahren 1843 bis 1851 gemachten Aufnahmen, von 1852 bis 1865 graviert im topographischen Bureau in Zürich (Wild-Karte).

Malerischer Plan der Stadt Zürich und ihrer Umgebungen. Herausgegeben von H. F. Leuthold, Zürich. Ca. 1850. 
3.2.2.2. Die Epoche der Dampfmaschine in Verkehr und Industrie (1860 - 1920)

Der stärkste Faktor für die gründliche Umwandlung der Siedlungsstruktur in der zweiten Phase der mesotechnischen Epoche ist die seit der Mitte des 19. Jahrhunderts erfolgende Anlage des Eisenbahnnetzes. Infolge der Standortvorteile, die es sowohl für Arbeits wie für Wohnplätze bot, richtete sich die Ueberbauung des Kantonsgebietes mehr und mehr nach diesem Netz aus. Mit dem neuen Verkehrsmittel im Zusammenhang (Verbilligung der Kohlentransporte) steht der Aufstieg der Dampfmaschine zur industriellen Hauptantriebskraft neben der Wasserturbine (die am Ende des 19. Jahrhunderts einsetzende Verwendung der Elektrizität verstärkt bis zum Beginn des neotechnischen Zeitalters lediglich die siedlungsgeschichtlichen Auswirkungen der Dampfmaschine). Damit fiel für die industrielle Agglomeration (Ballung industrieller Arbeitsplätze) nicht nur eine natürliche Schranke, welche am jeweiligen Standort ihre maximale Ausdehnung bisher limitiert hatte, sondern seit dem letzten Viertel des 19. Jahrhunderts entstanden industrielle Agglomerationen in günstiger Eisenbahnverkehrslage überhaupt unabhängig vom Gewäs sernetz (z.B. Oerlikon, Abb. 2). - Mit der Anlage des Eisenbahnnetzes und dem Aufkommen hydrographisch unabhängiger industrieller Antriebskräfte erfassen wir die hauptsächlichen Determinanten des Siedlungsbildes im Kanton Zürich am Ende der mesotechnischen Epoche. Aus diesen beiden Komponenten gingen die industriellen, demographischen und baulichen

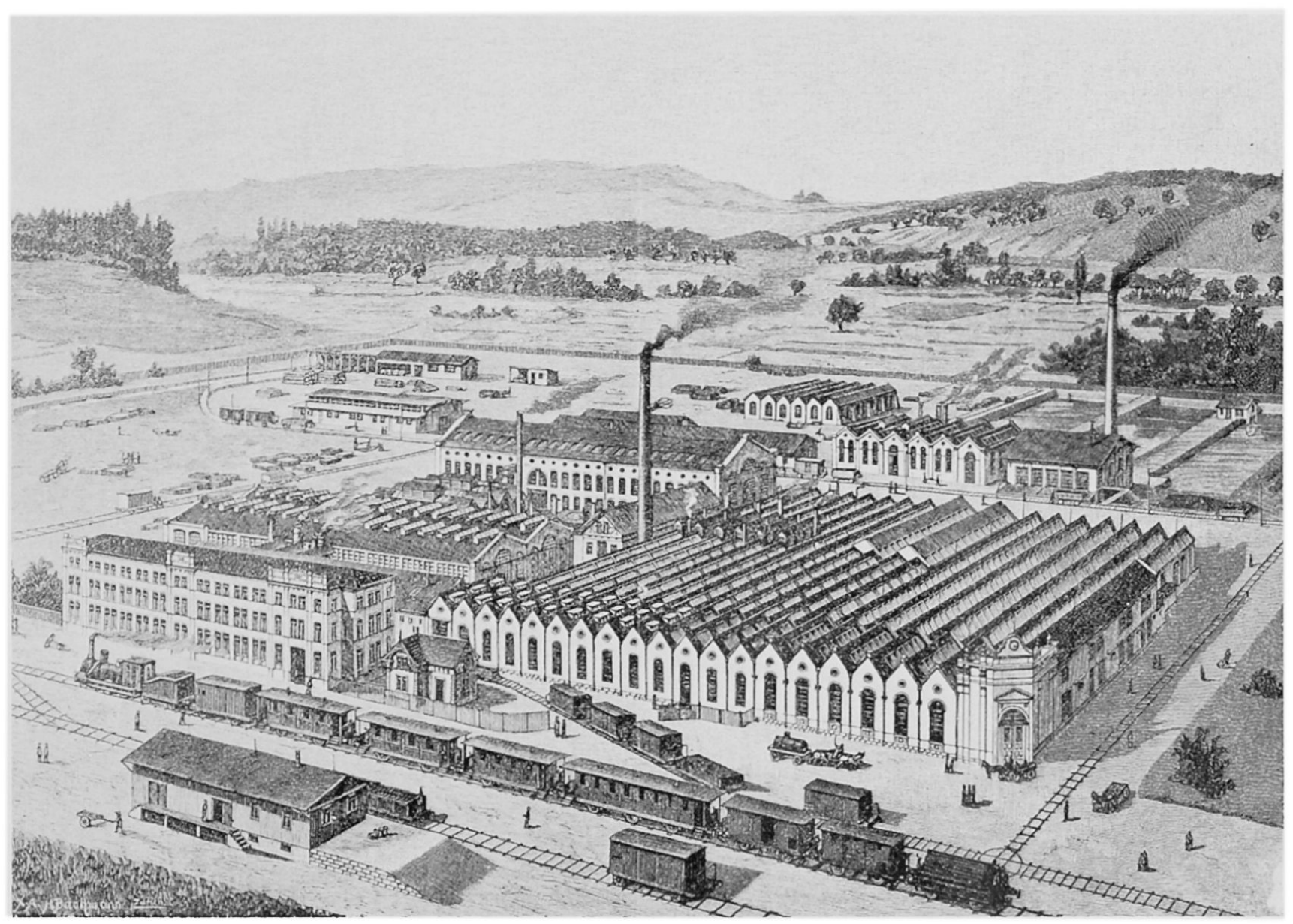

Abb. 2: Maschinenfabrik Oerlikon 1896 (Zentralbibliothek Zürich, Graphische Sammlung). 
Agglomerationszonen hervor, in erster Linie die kompakten Ballungen um die Städte Zürich und Winterthur, aber auch die noch diskontinuierlichen, von Gebieten stagnierender Besiedlung unterbrochenen Verdichtungszonen in den Talachsen von Haupttälern überhaupt.

Der Agglomeration steht die Deglomeration (Stagnation oder Rückbildung der Besiedlung) in jenen Gebieten gegenüber, wo Fabrikindustrie nicht in strukturrelevantem Masse Eingang fand (besonders Weinland und Rafzerfeld). Deglomerationsgebiete sind im Kanton Zürich am Ende der mesotechnischen Epoche die oberen Höhenstufen überhaupt sowie die Talachsen der Nebentäler ohne Eisenbahnanschluss (Abb. 3). - Am Ende der mesotechnischen Epoche erscheint als Gestaltungsprinzip der Siedlungsstruktur im Kanton Zürich nicht mehr der für die paläotechnische Epoche kennzeichnende Siedlungsraster; sie wird nun in erster Linie durch den Gegensatz zwischen den agglomerativen und den deglomerativen Zonen bestimmt, wobei in den agglomerativen Gebieten der Siedlungsraster selbst durch die infolge der Siedlungsverschmelzungen neuentstandenen Siedlungskomplexe grossenteils überformt und aufgesogen worden ist.

Mit diesem Strukturverlust geht eine Verwischung der Unterschiede zwischen den Siedlungstypen einher: In der zweiten Phase der mesotechnischen Epoche entsteht als neuer Siedlungstypus der Industrieort, eine Mischform aus den beiden überkommenen. Haupttypen "Dorf" und "Stadt". - Auch beginnt im mesotechnischen Zeitalter die Aufhebung der Identität zwischen zentralem Ort und zentraler Ortschaft: Können für das Paläotechnikum die auch baulich von der umliegenden Landschaft klar abgehobenen Städte noch je gesamthaft als zentrale Orte gelten, so ist das für das Ende der mesotechnischen, Epoche, vor allem hinsichtlich der Grossstadt Zürich, nicht mehr möglich; zentrale Orte sind hier nur das Hauptzentrum (City) und die Subzentren (z.T.Quartierzentren); die dazwischen liegenden Wohnund Industrieviertel dagegen können als disperse Zonen angesehen werden.

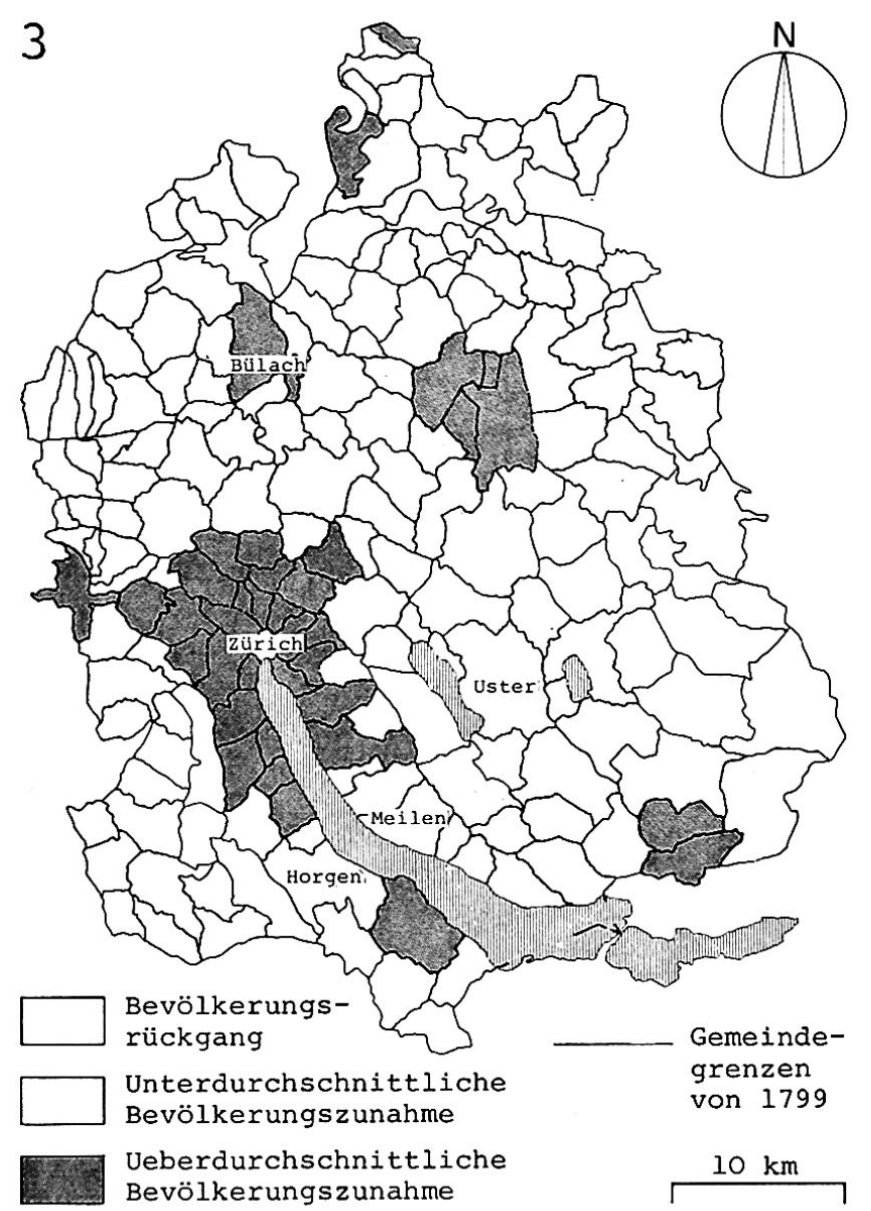

Abb. 3: Agglomeration und Deglomeration der Bevölkerung 1799-1920.

Quelle: W. Raths: Die Bevölkerung des Kantons Zürich seit Ende des 18. Jahrhunderts; Zürich, 1949.

3. 2. 3. Neotechnisches Zeitalter (seit ca. 1920)

3.2.3.1. Faktoren und Verlauf der ungelenkten Besiedlung

Nach 1900 begannen eine Reihe Erfindungen aus der 2. Hälfte des 19. Jahrhunderts allgemein verwertet $z u$ werden: die Fernübertragung elektrischer Energie, welche den Elektromotor zur industriellen Hauptantriebskraft werden liess, der Explosionsmotor, das Telephon sowie der radioelektronische drahtlose Nachrichtenverkehr. Die daraus hervorgehende neue Technologie verkehrte das agglomerative Gestaltungsprinzip der mesotechnischen Epoche in sein Gegenteil: die 
fast völlige Standortunabhängigkeit der Ar beits - und Wohnstätten von allen Determinanten, mit Ausnahme von Bodengestalt und geschichtlicher Vorformung. Die Siedlungsgeschichte schreitet also von der paläotechnischen gerasterten Siedlungsstruktur (Hauptdeterminante: Bodenform und Bodenqualität) über die mesotechnische agglomerativ-deglomerative (Hauptdeterminante: Eisenbahnnetz) zur neotechnischen Struktur, die in ebenem Gelände, unter Ausklammerung der historischen Vorformung, überhaupt nicht determiniert ist: die neotechnische Siedlungsstrukturist an sich ubiquitär (d.h. die Wohn- und Arbeitsstätten verteilen sich in einem bestimmten geographischen Raum beliebig).

Neben der Naturdeterminante des im Kanton Zürich bewegten Reliefs tritt aber nun die historische Vorformung des Siedlungssystems als starker Deviationsfaktor der möglichen Entwicklung zu einer ubiquitären Struktur in Erscheinung. Waren, aufgrund dieser Vorformung, schon in der mesotechnischen Epoche die zentralen Orte Zürich und Winterthur, in langfristig historisch gewordenem Abstand, die Hauptknotenpunkte des Eisenbahnnetzes geworden, denen sich die grössten Bevölkerungs - und Siedlungsballungen anlagerten, so verstärkte sich im neotechnischen Zeitalter das Gewicht dieser städtischen Zentren - wenigstens zunächst - noch.Die Gründe dafür liegen in einer relativen Gewichtsverlagerung der verschiedenen Wirtschaftssektorren sowie einer entsprechenden Verschiebung in der beruflichen Gliederung der Bevölkerung und in deren soziokultureller Veränderung. Gab um die Jahrhundertwende noch der sekundäre Wirtschaftssektor (vorab Industrie), dem die Mehrzahl der Berufstätigen angehörte,dem Kanton $Z \ddot{u}-$ rich sein Gepräge, wo wurde er seit Beginn der neotechnischen Epoche zunehmend vom tertiären Sektor (Dienstleistungen, Verwaltung, auch privater Unternehmungen) aus seiner vorherrschenden Stellung verdrängt, nicht zuletzt wegen der beschleunigten Produktivitätssteigerung in der Industrie (u. a. durch Automation), welche Arbeitskräfte zugunsten des tertiären Wirtschaftssektors freisetzte, und heute gehört die Mehrzahl der Berufstätigen diesem Sektor an: An die Stelle der Arbeiterschaft mit ihrer aus städtischen und ländli- chen Elementen gemischten Subkultur treten die Angestellten, ein neuer Mittelstand mit einer auf metropolitane Leitvorstellungen ausrichteten Kultur und Lebensweise, als quantitativ dominierende Sozialschicht. Die Betriebe des gehobenen Dienstleistungssektors (Banken, Finanzgesellschaften, Versicherungen, Gross handelsfirmen, Bildungs - und Forschungsinstitute) hatten sich schon im Mesotechnikum in den zentralen Orten (Zürich und Winterthur in historisch gegebenem Abstand) niedergelassen und expandierten vor allem dort. $\mathrm{Zu}$ ihnen gesellten sich im Neotechnikum, wegen der Fühlungsvorteile, eine Reihe weiterer Dienstleistungs - und Verwaltungsbetriebe, die expandierende Bürokratie des Sozialstaates, die Apparate der Wirtschaftsverbände, die Zentren der Massenmedien, die Administrations- und Fors chungszentren multinationaler Industriegesellschaften etc.

Infolge dieser Entwicklungen nahm die Zahl der Arbeitsplätze in der Stadt Zürich, in zweiter Linie auch in Winterthur, rapid zu. Die aufgrund des sozio-kulturellen Wandels urbanisierte Bevölkerung siedelte sich vor allem in den stadtnahen Gemeinden an, und im Anschluss an die Stadt Zürich expandierte - mit gleichzeitiger innerer Verdichtung - eine ausgedehnte metropolitane Siedlungsregion. In dieser Siedlungsregion - und in geringerem Masse auch im übrigen Kantonsgebiet - beginnen sich nun Entwicklungsrichtungen durchzusetzen, die im Gegensinne zum Mesotechnikum verlaufen: Ueberbauung auch von Hängen, Hochterrassen (Abb. 4), Hügelkuppen und Bergrücken (Hauptbeispiel: Forch - Pfannenstielgebiet). Bezeichnend für das Neotechnikum ist das Ueberhandnehmen ubiquitär-ungerichteter Streusiedlung mit z.T. grossflächigen Siedlungskonglomeraten (Abb. 5). Das neotechnische Zeitalter hat auch einen neuen Typus zentraler Orte hervorgebracht: Shopping Centers mit Anschlüssen zu Autobahnen, welche historisch gewordene Stadtregionen miteinander verbinden (Spreitenbach, Glatt).

3.2. 3. 2. Einflüsse der räumlichen Planung Die mit dem Liberalismus nach dem Untergang des Ancien Régime aufkommende extreme Baufreiheit hatte schon im Mesotechnikum und zunehmend im Neotechnikum Miss- 


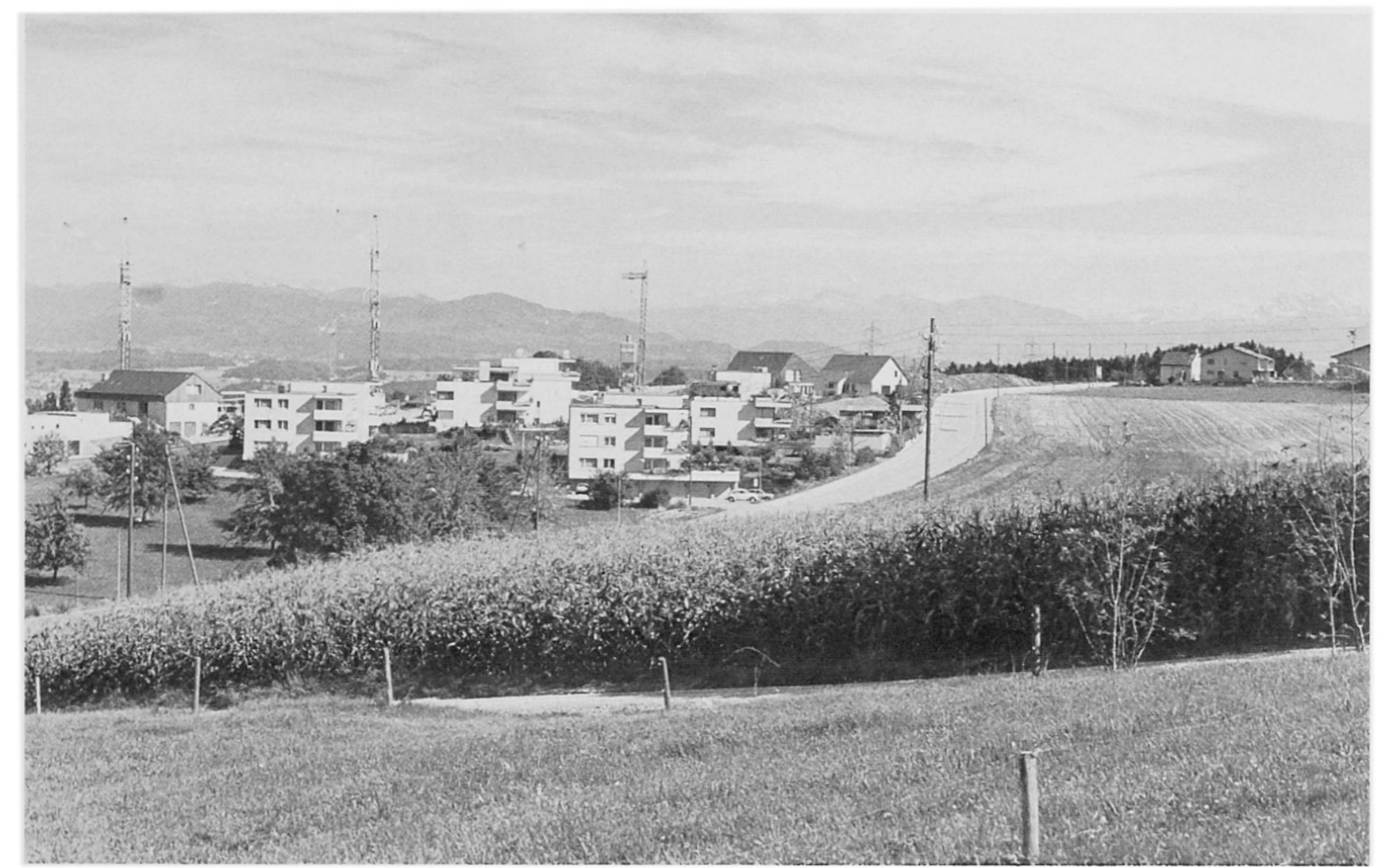

Abb. 4: Beispiel einer neotechnischen Hochterrassensiedlung am Pfannenstiel.

stände hygienischer, ästhetischer und sozialpsychischer Art zur Folge. Erste Ansätze, ihnen entgegenzuwirken, waren die kantonalen Baugesetze von 1863 und 1893. War der Hauptinhalt dieser Gesetze noch bau- und sanitätspolizeilicher Natur, so wurde durch sie den Gemeinden doch die Kompetenz erteilt, strengere Bauordnungen zu erlassen, welche - wie bereits die Bauordnungen der Stadt Zürich von 1901 und 1912 - die Realisierung ortsplanerischer und städtebaulicher Grundsätze ermöglichten. Das Postulat einer Landes - und Regionalplanung wurde aber erst in der neotechnischen Epoche erhoben. Teilrevisionen des Baugesetzes von 1893 schufen dafür die rechtlichen, freilich unzulänglichen Voraussetzungen. Eine vom Regierungsrat beauftragte Expertenkommission erarbeitete in der Folge die Grundsätze und Probleme einer Gesamtplanung im Kanton Zürich. In ihrem 1960 veröffentlichten Bericht empfahl diese
Kommission eine Variante "Regionalzentren" (dezentralisierte Konzentration) als Leitbild der Besiedlung. Von diesem Konzept ging der kantonale Gesamtplan aus, dessen Ausarbeitung 1963 beschlossen und dem neugeschaffenen Amt für Regionalplanung (heute Amt für Raumplanung) übertragen wurde. - In der projektierten Siedlungsgeschichte werden die Auswirkungen der Ortsplanung sowie Realisiemungen regionalplanerischer Konzepte an signifikanten Beispielen nachzuweisen sein. Ebenso werden bauliche Entwicklungen aufzuzeigen sein, die im Gegensatz zu den offiziellen Konzepten verlaufen sind. 1975 wurden durch das neue Bau- und Planungsgesetz ausreichende rechtliche Voraussetzungen geschaffen, die ungeordnete Siedlungsentwicklung und Bodennutzung in den einzelnen Regionen und im Gebiet des Kantons Zürich überhaupt in dem Masse zu lenken, als es das öffentliche Interesse erfordert. (Subsi- 


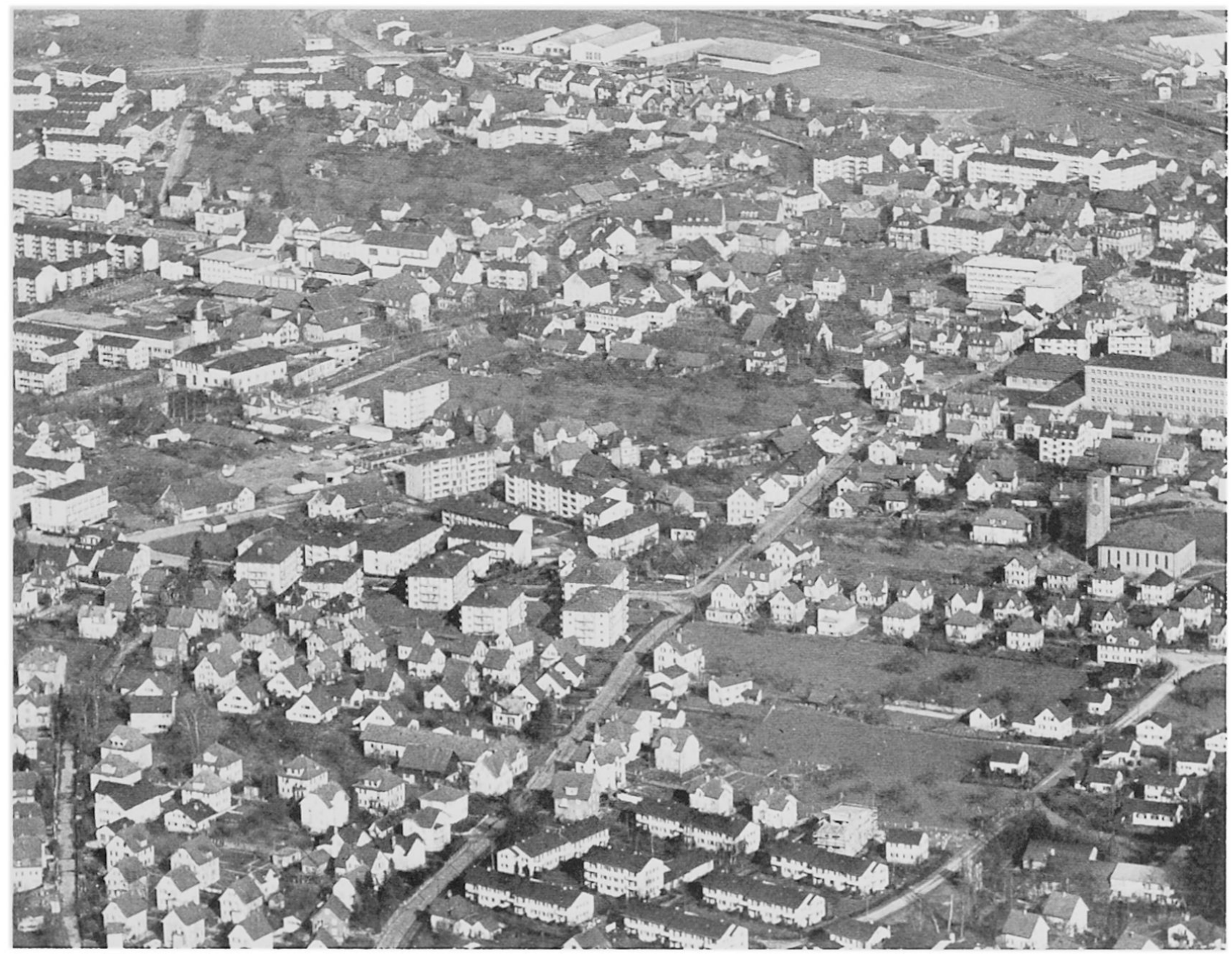

Abb. 5: Beispiel eines Siedlungskonglomerats im Limmattal (Photoswissair).

diäre Planungstätigkeit des Bundes: Gründung des ORL-Institutes an der ETHZ 1961 -Verfassungsartikel 1969 - Ausführungsgesetzgebung: 1972 Bundesbeschluss, womöglich vor 1979 Raumplanungs gesetz. )

3. 2. 4. Nachtechnisches Zeitalter?

Ob eine solche Epoche überhaupt als eine Möglichkeit künftiger Geschichte aufgefasst werden kann, wird eine gründliche Erörterung abklären müssen. Voraussetzung einer nachtechnischen Epoche wäre nicht etwa das Verschwinden der Technik, sondern im Gegenteil ihre Perfektionierung bis zu einem Grade, wo sie sich als Determinante der Siedlungsgeschichte - und vielleicht der Geschichte überhaupt - selber aufhebt. Die Sachzwänge, die aus der Entwicklung der Technik erfolgten, fielen dahin; Art und Ausmass der für die Daseinsbewältigung anzuwendenden Techniken würden ins Belieben der Gesellschaft gestellt. Die Annahme, aus der Perfektionierung der Technik werde zwangsläufig ein "nachtechnisches Zeitalter" mit einem radikalen Gestaltwandel der Ge schichte hervorgehen, wäre eine Spekulation im Sinne eines monokausalen Determinismus und im Widerspruch mit der historischen Erfahrung. Die Perfektion der Produktionsmittel schafft lediglich die notwendige materielle Voraussetzung zu einem solchen Gestaltwandel, nämlich zur Realisierung jenes Humanitätside- 
als, das sich zeitlich parallel zur technischen Revolution entfaltet. Können heute schon Indizien beobachtet werden, welche die Verwirklichung dieses Leitbildes - optimale Selbstverwirklichung des Einzelnen in einer solidarischen Gesellschaft - als Möglichkeit der Zukunft erscheinen lassen? Es dürfte eine ganze Reihe solcher Ansätze festzustellen sein; hier seien nur zwei genannt: Einmal ist die politische und gesellschaftliche Emanzipation der Frau in Betracht zu ziehen, die ja erst in den 70 er Jahren die Gesamtheit des weiblichen Bevölkerungsteils zu erfassen begann. Könnten dadurch in Zukunft die geschichtsbestimmenden Faktoren -nicht zu letzt die Technologie im Bewusstsein der Gesellschaft nicht völlig andere Wertungen erfahren und somit auch anders sich auswirken als bis anhin, zumal in den Wohn- und Siedlungsformen? - Zum zweiten sei auf die Revolte eines Teils der jungen Generation um 1970 hingewiesen, die in ihrer Substanz ein Aufstand gegen die neotechnische Gesellschaft, deren Wertsysteme und Leitvorstellungen gewesen sein dürfte. War sie lediglich eine Episode im Sinne eines psychologisch begründeten Generationenkonflikts, eine Auflehnung als blosse Negation und ohne geschichtsbestimmende Bedeutung? Oder liegen in ihr erste Ansätze zu neuen Lebensformen und damit auch zu neuen Wohn- und Siedlungsweisen der Zukunft?

Beim gegenwärtigen Stand des Wissens kann kein "nachtechnisches Zeitalter" mit bestimmten Gesellschafts-, Wirtschafts - und Siedlungsformen prognostiziert werden. Der heutige Stand der Technik und die Möglichkeit von Bewusstseinsveränderungen in der Gesellschaft, die sich vielleicht jetzt schon abzeichnen, können aber dazu ermutigen, Entwicklungsvarianten einer nachtechnischen Epoche mit entsprechenden Strukturvarianten der Besiedlung zu skizzieren, wobei die Unterscheidung zwischen wahrscheinlichen und unwahrscheinlichen Verläufen anzustreben wäre.

1) Begriffe und Begriffsdefinitionen sind vom Verfasser für die vorliegende Arbeit teilweise in bewusster Abweichung von üblichen Formulierungen festgelegt worden.

\section{Literaturbesprechung}

MAIER Jörg: Zur Geographie verkehrsräumlicher Aktivitäten. Theoretische Konzeption und empirische Ueberprüfung an ausgewählten Beispielen in Südbayern. Münchner Studien zur Sozial- und Wirtschaftsgeographie, Bd. 17. 192 S., 28 Karten, 30 Abb., 9 Tab. Verlag Michael Lassleben, Kallmuinz/Regensburg 1976. DM 42.20.

Die umfangreiche Habilitationsschrift des Münchner Geographen Jörg MAIER gliedert sich in drei Teile: Im ersten Teil der Untersuchung wird der empirisch-theoretische Bezugsrahmen vorgestellt. Dabei wird besonderes Gewicht der Verankerung der vorliegenden Arbeit im sozialgeographischen Konzept beigemessen sowie der für die Analyse verkehrsräumlicher Aktivitäten notwendigen interdisziplinären Zusammenarbeit.

Als Testgebiet für seine Untersuchungen wählte MAIER Suidbayern. Dieses Gebiet zeichnet sich durch ein breites Spektrum unterschiedlicher Gemeindetypen aus. Da eine Totalerhebung ausgeschlossen war, wurde eine Stichprobenerhebung in 40 ausgewählten regionalen und innerstädti- schen Testgebieten durchgeführt. Im zweiten Teil der Arbeit wird dieser methodisch-empirische Ansatz der Untersuchung diskutiert.

Der dritte Teil der Untersuchung trägt die Ueberschrift 'Analyse der verkehrsräumlichen Verhaltensmuster, der Reichweitensysteme und der Verkehrsmittelwahl in ihrer regional- und gruppenspezifischen Differenzierung'. MAIER unterscheidet dabei zwischen erwerbs- oder arbeitsorientierten, versorgungsorientierten, freizeitorientierten und ausbildungsorientierten Verkehrsbewegungen. Den Abschluss der Untersuchung bildet die Bestimmung und regionale Verteilung von Gruppen gleichartigen verkehrsräumlichen Verhaltens. Dabei konnte zwischen elf Grundtypen unterschieden werden.

Es bleibt zu hoffen, dass diese interessante Arbeit mit ihren instruktiven Karten den Anstoss gibt, sich auch in der Schweiz vermehrt mit verkehrsgeographischen Problemstellungen auseinanderzusetzen.

GH $3 / 77$

Hans Elsasser 